\title{
Una nueva aproximación metodológica basada en redes conceptuales y redes probabilísticas para evaluar la provisión de servicios de los ecosistemas
}

\author{
Florencia Rositano $^{\bowtie}$ \& Diego O. Ferraro
}

IFEVA. Universidad deBuenosAires. CONICET. DepartamentodeProducción Vegetal. Cátedra deCerealicultura. Facultad
de Agronomía. Buenos Aires, Argentina.

\begin{abstract}
Resumen. En los últimos años han surgido varias herramientas para evaluar la provisión de servicios de los ecosistemas (SE) desde un punto de vista ecológico. No obstante, la complejidad de los SE desalienta los intentos de adoptar una única aproximación metodológica. El objetivo de este trabajo consistió en evaluar un nuevo marco de análisis de la provisión de SE, sobre la base de redes conceptuales y redes probabilísticas. Para cumplir con el objetivo se describió el desarrollo de una red conceptual y se representó el conjunto de variables que determinan la provisión de ocho SE de la Región Pampeana. Luego, se parametrizó esa red mediante una metodología probabilística conocida como Redes Bayesianas, para ser, después, aplicada a tres zonas agrícolas pampeanas. Por último, se plantearon ventajas y desventajas de este nuevo marco de análisis mediante una comparación con otras aproximaciones para el estudio de la provisión de SE, desarrolladas en Argentina y en otras partes del mundo, tales como InVEST, RIOS, ARIES y ECOSER. El enfoque aquí presentado puede ser útil para: a) evaluar la sustentabilidad de los agroecosistemas pampeanos desde una dimensión ecológica, y/o b) asistir a los tomadores de decisión (i.e., productores y asesores agropecuarios) para implementar estrategias sustentables de uso de la tierra.
\end{abstract}

[Palabras clave: Redes Bayesianas, redes conceptuales, servicios de los ecosistemas, conocimiento experto]

\begin{abstract}
A new methodological approach based on conceptual and probabilistic networks to assess ecosystem services provision. In the last few years, there have been an increasing number of tools to environmentally assess ecosystem services (ES) provision. However, its complexity discourages attempts to adopt a single methodological approach. Then, the aim of this study was to assess a new framework to analyze ES provision, based on conceptual and probabilistic networks. First, the new methodological approach was described. This approach consisted of a conceptual network representing those variables that determine eight ES provided by the Pampa region. The conceptual network was parameterized using a probabilistic methodology known as Bayesian networks, and then was applied to three Pampean agroecosystems. Finally, the new analytical framework was compared with other national and international approaches that assess ES provision, such as InVEST, RIOS, ARIES and ECOSER. The approach presented here could be helpful to: a) assess the sustainability of Pampean agroecosystems from an ecological dimension, and/or b) to assist decision makers (i.e., farmers and their advisors) in the implementation of sustainable land use strategies.
\end{abstract}

[Keywords: Bayesian Networks, conceptual networks, ecosystem services, expert knowledge]

\section{INTRODUCCIÓN}

Existen aspectos del funcionamiento de los ecosistemas que actualmente se analizan por medio de abordajes cualitativos (Lamanda et al. 2012). Las metodologías cualitativas permiten integrar interpretaciones lógicas de información difícil de cuantificar, y observar las relaciones causales entre procesos ecosistémicos (Busch et al. 2012). Un ejemplo es la representación de los factores bióticos (i.e., ambiente) y abióticos (i.e., prácticas de manejo agrícola) en modelos conceptuales para explicar la variabilidad en el rendimiento de los cultivos (Rapidel et al. 2006). La ventaja principal de la representación conceptual es su capacidad para indicar tendencias, potencialidades y

Editor asociado: Pedro Laterra

rositano@agro.uba.ar conflictos en los ecosistemas, y para evitar el riesgo de sobreinterpretar datos cuantitativos insuficientes (Busch et al. 2012). También permite reconocer rápidamente los efectos potenciales de la actividad humana dentro de los sistemas, y estimula la reflexión del rol de la humanidad sobre estos efectos. Por lo tanto, se considera que la modelización conceptual es una estrategia promisoria para representar la complejidad de los ecosistemas.

Por otro lado, los cambios constantes a los que están expuestos los ecosistemas, y su capacidad para proveer servicios, hacen necesario que su evaluación no sólo proporcione una instantánea de su estado actual, sino también que permita anticipar situaciones futuras. En este sentido, se

Recibido: 10 de agosto de 2015

Aceptado: 17 de noviembre de 2016 
plantea la necesidad de usar herramientas que lidien tanto con la complejidad como con la incertidumbre inherente a estos sistemas. Walker y colaboradores (2003) distinguen tres dimensiones para caracterizar la incertidumbre directamente relacionadas con la modelización de los ecosistemas: a) la localización de la incertidumbre (e.g., dónde se manifiesta la incertidumbre dentro de la complejidad del modelo), b) el nivel de incertidumbre (e.g., dónde se manifiesta la incertidumbre en el rango propuesto de conocimiento), y c) la naturaleza de la incertidumbre (donde se explica si la incertidumbre responde a la imperfección del conocimiento o si es propia de la variabilidad del fenómeno bajo estudio). Una alternativa para reducir el efecto de la incertidumbre en la modelización de los ecosistemas es la incorporación de conocimiento experto (Cornelissen et al. 2003).

En la actualidad, las Redes Bayesianas (RB) son un método cada vez más popular para modelar dominios inciertos y complejos (Ellison et al. 1998; Dorner et al. 2007; Chen and Pollino 2012) como los ecosistemas (Uusitalo 2007). Se debe considerar a las RB como un integrador del conocimiento y no como un reemplazo de otros modelos en uso (Borsuk et al. 2004). Tienen la habilidad de capturar los aspectos estructurales de un problema de decisión, así como de servir de estructura para su eficiente análisis cuantitativo (Dorner et al. 2007). En términos generales, son una representación gráfica de un conjunto de variables con una distribución probabilística (van der Gaag and Helsper 2002). Su resultado evalúa cuán probables son los eventos y cómo estas probabilidades cambian con las intervenciones externas (Pollino et al. 2007). Su principal ventaja es la obtención de modelos explícitos, consistentes y reproducibles, al mismo tiempo que su lógica interna puede ser modificada según la información disponible (e.g., conocimiento experto, bases de datos) en cualquier momento (López Puga et al. 2007; Low Choy et al. 2009). En este sentido, las RB permiten cuantificar tanto la subjetividad de la información proporcionada como la incertidumbre en las estimaciones de los parámetros (Ascough et al. 2008). Sin embargo, presentan ciertas dificultades inherentes como, por ejemplo, su capacidad limitada para modelar sistemas dinámicos (en tiempo o espacio) (Landuyt et al. 2014) dado que los modelos desarrollados deben ser acíclicos (i.e., sin relaciones circulares para evitar que las probabilidades ciclen indefinidamente). Si bien algunos estudios recientes han utilizado las $\mathrm{RB}$ con el fin de examinar cuestiones pertinentes a los procesos y las funciones de los ecosistemas (Haines-Young 2011; Grêt-Regamey et al. 2012), es necesario seguir trabajando con esta metodología para superar las actuales limitaciones que presenta la evaluación de sistemas espacio-temporales complejos (Smith et al. 2011).

En función de estos antecedentes, el objetivo del presente trabajo consiste en evaluar un nuevo marco de análisis de la provisión de servicios de los ecosistemas (SE), sobre la base de redes conceptuales y redes probabilísticas (i.e., RB), recientemente aplicado por Rositano and Ferraro (2014). Con ese fin, primero se describe la nueva aproximación metodológica y luego se comparan sus ventajas y desventajas en relación a otras herramientas disponibles.

\section{ESTUDIO DE CASO: REDES

CONCEPTUALES Y REDES

\section{PROBABILÍSTICAS PARA EVALUAR}

\section{LA PROVISIÓN DE SERVICIOS EN}

\section{AGROECOSISTEMAS PAMPEANOS}

La evaluación dela provisión deSEse presenta como un nuevo enfoque para estudiar la sustentabilidad de los agroecosistemas ya que representa su funcionamiento desde un punto de vista sistémico al incluir tanto la condición biofísica como la dimensión utilitaria de estos ecosistemas (Müller 2005). Además, los SE permiten evaluar los cambios que generan las acciones humanas en los ecosistemas y resolver los conflictos que surgen por los distintos usos de la tierra (Vihervaara et al. 2009). En este sentido, Rositano and Ferraro (2014) desarrollaron un marco de análisis con el objetivo de evaluar los cambios en la provisión de SE como consecuencia de la variabilidad ambiental y de las prácticas de manejo agrícola en los agroecosistemas pampeanos. Este marco de análisis se basó en dos herramientas capaces de lidiar con el problema de la complejidad de los ecosistemas y con su incertidumbre: las redes conceptuales y las redes probabilísticas. Este nuevo enfoque puede ser de utilidad para: a) evaluar la sustentabilidad de los agroecosistemas pampeanos desde una dimensión ecológica, y/o b) asistir a los tomadores de decisión (i.e., productores y asesores agropecuarios) en la implementación de estrategias sustentables de uso de la tierra. De aquí en adelante, este marco analítico será reconocido como $\mathrm{RC}+\mathrm{RB}$ en relación a las metodologías que se usaron: 


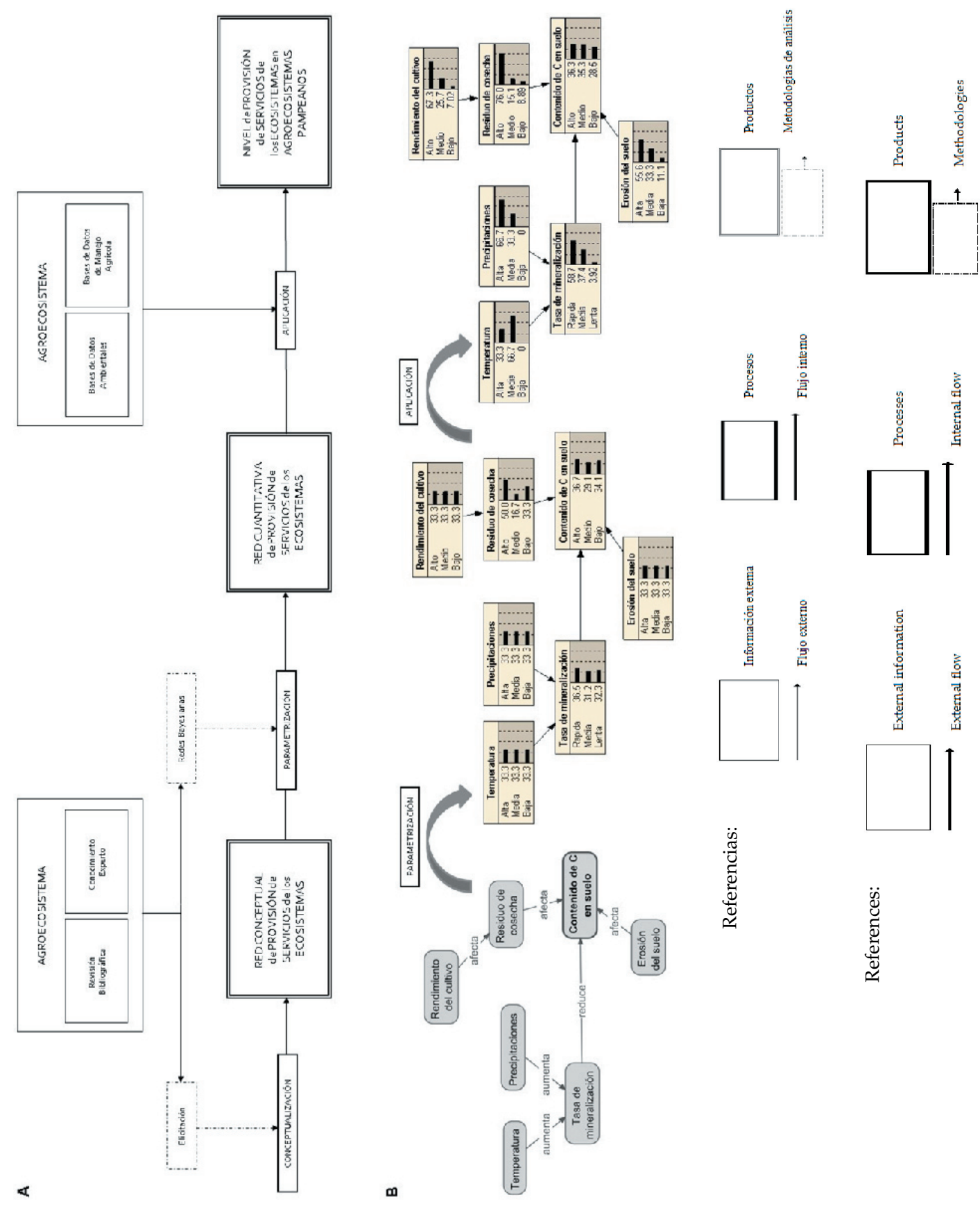

Figura 1. A) Representación esquemática del proceso lógico de desarrollo del nuevo marco analítico de evaluación de la provisión de servicios de los ecosistemas en los agroecosistemas pampeanos basado en redes conceptuales y redes probabilísticas. En el esquema se representan los pasos principales junto con la metodología de análisis y la información externa necesaria para obtener los productos principales de cada etapa. B) Ejemplo de los productos principales de cada etapa del nuevo marco analítico en base al servicio del ecosistema balance de C del suelo. En la etapa de parametrización sólo se presenta el resultado de la elicitación del conocimiento experto en aquellas variables dependientes de una o más variables. La etapa de aplicación se ejemplifica con información ambiental y productiva correspondiente a la zona sur de Entre Ríos para el cultivo de maíz durante la campaña 2000/2001.

Figure 1. A) Schematic representation of the logical process for developing a new analytical framework for assessing ecosystem services provision in Pampean agroecosystems based on conceptual and probabilistic networks. Main steps are represented along with the methodology and external information necessary to obtain the main products of each stage. B) Example of main products of the new analytical framework based on soil C balance ecosystem service. In parameterisation stage, only results from expert knowledge elicitation in those variables dependent of one or more variables are shown. The applying step is exemplified with environmental and productive information of South of Entre Rios for maize during 2000/2001. 
redes conceptuales y Redes Bayesianas. El desarrollo de $\mathrm{RC}+\mathrm{RB}$ tuvo tres etapas (Figura 1).

En primer lugar, se realizó una red conceptual en la que se representó de manera cualitativa el conjunto de variables ambientales y productivas que determinan la provisión de ocho SE en la Región Pampeana (Figura 1A). Los SE seleccionados fueron: 1) balance de carbono (C) del suelo (Figura 1B), 2) balance de nitrógeno $(\mathrm{N})$ del suelo, 3) mantenimiento de la estructura del suelo, 4) balance hídrico del suelo, 5) control de la emisión de $\mathrm{N}_{2} \mathrm{O}, 6$ ) regulación de adversidades bióticas, 7) control de contaminación del agua subterránea, y 8) mantenimiento de la riqueza de especies. Esta lista se basa sobre un concepto de SE que no sólo incluye los atributos y procesos de los ecosistemas que soportan SE, sino también los servicios en sentido más estricto. La red conceptual fue el resultado de un proceso de revisión bibliográfica y de obtención de conocimiento experto mediante entrevistas. Los expertos considerados fueron investigadores involucrados con diversas áreas de los sistemas agrícolas (e.g., fertilización de cultivos, contaminación ambiental por fertilizantes, dinámica de nutrientes, calidad y contaminación del agua subterránea, fertilidad del suelo, ecofisiología de malezas). Los investigadores fueron seleccionados dentro del campo académico de la Facultad de Agronomía de la UBA (FAUBA) y también de otras universidades nacionales (i.e., Universidad Nacional de Mar del Plata, Universidad Nacional de San Luis) e instituciones (i.e., Instituto Nacional de Tecnología Agropecuaria, International Plant Nutrition Institute). El panel de expertos estuvo compuesto por 20 investigadores.

En segundo lugar, se seleccionaron cuatro sub-redes desprendidas de la red conceptual general, con el objetivo de parametrizarlas mediante RB (Figura 1A). Estas sub-redes fueron: a) balance de $\mathrm{C}$ del suelo (Figura 1B), b) balance de $\mathrm{N}$ del suelo, c) control de emisión de $\mathrm{N}_{2} \mathrm{O}, \mathrm{y}$ d) control de la contaminación del agua subterránea. Antes del proceso de parametrización fue necesario determinar el número de estados (alto, medio y bajo) de cada variable. Los estados son "clusters" o grupos delimitados por intervalos o rangos de las variables bajo estudio. El número de estados depende de la información que se desee transmitir y de los posibles valores que pueda llegar a tomar la variable (Dlamini 2010). En este caso, las variables presentaron entre dos y tres estados (ver Material Suplementario). La parametrización tiene como finalidad obtener las probabilidades condicionales a partir de una estructura conceptual (Bressan et al. 2009). Aquellas variables determinadas por una o más variables están caracterizadas por una tabla de probabilidad condicional que representa la combinación de todos los estados de las variables de las cuales depende junto con sus valores probabilísticos. Por su parte, las variables de entrada tienen distribuciones probabilísticas marginales que representan la frecuencia de cada estado (Chen and Pollino 2012) (Figura 1B). Las tablas de probabilidad condicional fueron el resultado de un proceso de obtención de conocimiento de un subconjunto de los expertos entrevistados en la etapa cualitativa. Tanto la RB para cada SE como las tablas de probabilidad condicional figuran en el Material Suplementario para que puedan ser utilizadas por futuros usuarios.

Finalmente, las variables de entrada de los modelos se cuantificaron con bases de datos ambientales y productivas (Figura 1A) provenientes de tres zonas agrícolas pampeanas con distintas características agroecológicas (norte de Córdoba, centro de Buenos Aires y sur de Entre Ríos). Las bases

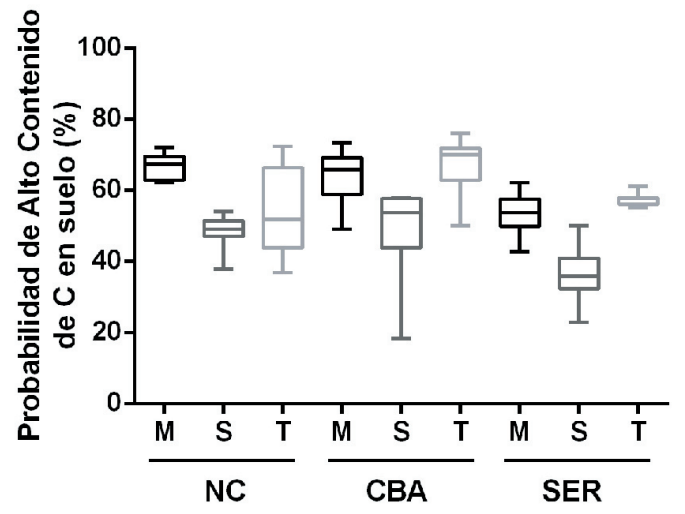

Figura 2. Respuesta probabilística de la variable respuesta alto contenido de $\mathrm{C}$ en suelo, correspondiente al servicio del ecosistema balance de $C$ del suelo, en relación a las tres zonas agrícolas bajo estudio. En cada caja se representan los cuartiles Q1 (25\% de los datos), Q2 ó mediana (50\% de los datos) y Q3 (75\% de los datos). Los bigotes representan los valores mínimo y máximo de cada situación. Referencias: $\mathrm{M}=$ maíz, $\mathrm{S}=$ soja, $\mathrm{T}=$ trigo, $\mathrm{NC}=$ norte de Córdoba, $\mathrm{CBA}=$ centro de Buenos Aires, SER=sur de Entre Ríos.

Figure 2. Probabilistic response of the response variable high $\mathrm{C}$ content in soil, from the soil $\mathrm{C}$ balance ecosystem service model, in relation to three agricultural areas under study. In each box, the quartiles Q1 (25\% of the data), Q2 or median ( $50 \%$ of the data) and Q3 (75\% of the data) are represented. The whiskers represent the minimum and maximum values for each situation. References: $\mathrm{M}=$ maize, $\mathrm{S}=$ soybean, $\mathrm{T}=$ wheat, $\mathrm{NC}=$ north of Córdoba, $\mathrm{CBA}=$ center of Buenos Aires, SER=south of Entre Ríos . 
de datos ambientales fueron provistas por el Servicio Meteorológico Nacional(SMN) y porel Instituto Nacional de Tecnología Agropecuaria (INTA), mientras que las bases de datos productivas se obtuvieron de la Asociación Argentina de Consorcios Regionales de Experimentación Agrícola (AACREA). De esta manera, se obtuvo el nivel de provisión de los cuatro SE seleccionados, para diez campañas agrícolas (2000/2001-2009/2010) y tres cultivos (trigo, maíz y soja). En la Figura 2 se muestra un ejemplo de los resultados obtenidos para el modelo balance de $C$ del suelo, cuya variable respuesta es contenido de $C$ en suelo, con tres estados: alto, medio y bajo (Figura 1B). Para hacer más legibles los resultados, sólo se muestra el estado de la variable de respuesta deseable en los agroecosistemas pampeanos: alto contenido de $\mathrm{C}$ en suelo (Rositano and Ferraro 2014). Las diferencias más marcadas se vieron entre cultivos. La soja presentó los valores más bajos de probabilidad en las tres zonas de estudio (Figura 2).

\section{RC+RB FRENTE A OTRAS HERRAMIENTAS PARA EVALUAR LA PROVISIÓN DE SERVICIOS DE LOS ECOSISTEMAS}

El marco de análisis descripto en Rositano and Ferraro (2014) se comparó con un conjunto de herramientas desarrolladas a nivel nacional e internacional (Tabla 1). Entre éstas se encuentran aquellas de amplia difusión internacional, como ARIES (Artificial Intelligence for Ecosystem Services) (Villa et al. 2009, 2014), InVEST (Integrated Valuation of Environmental Services and Tradeoffs) (Kareiva et al. 2011; Tallis et al. 2013) y RIOS (Resource Investment Optimization System) (Vogl et al. 2013). En el ámbito nacional, se seleccionó una herramienta desarrollada por Laterra y colaboradores $(2015,2016)$ denominada ECOSER. A continuación, se presenta la comparación en base a seis criterios de evaluación.

\section{Evaluación de un conjunto de SE}

Las cinco aproximaciones evalúan un amplio grupo de SE. InVEST, ARIES, ECOSER y RC+RB analizan aquel SE directamente relacionado con el ciclado del $\mathrm{C}$ a diferencia de RIOS que únicamente evalúa los SE provistos por cuencas. Todos se enfocan en un SE directa o indirectamente relacionado con el ciclo del agua.

\section{Metodología de análisis}

La combinatoria de metodologías de análisis es amplia. ECOSER se compone de una integración de modelos, reglas e indicadores para evaluar y mapear la provisión de SE y la vulnerabilidad ambiental (Laterra et al. 2015). InVEST usa funciones simples de producción de la cobertura de la tierra y los atributos asociados (Tallis et al. 2013). RIOS se basa, por un lado, en ecuaciones e índices que se conjugan para construir modelos de impacto que se utilizan en el análisis diagnóstico para seleccionar los portafolios de inversión y, por otro lado, en modelos provenientes de InVEST utilizados para estimar el retorno de SE en la inversión tras la ejecución del portafolio (Vogl et al. 2013). ARIES combina ontologías (i.e.,

Tabla 1. Tabla integradora comparativa de cinco aproximaciones para evaluar la provisión de servicios de los ecosistemas en base a seis criterios de evaluación. Referencias: $\mathrm{SE}=$ servicio del ecosistema, ECOSER=Ecosystem Services, InVEST=Integrated Valuation of Environmental Services and Tradeoffs, ARIES=ARtificial Intelligence for Ecosystem Services, RIOS=Resource Investment Optimization System, RC+RB=redes conceptuales + Redes Bayesianas.

Table 1. Integrative comparative table of five approaches used to assess ecosystem services provision based on six evaluation criteria. References: SE=ecosystem service, ECOSER=Ecosystem Services, InVEST=Integrated Valuation of Environmental Services and Tradeoffs, ARIES=ARtificial Intelligence for Ecosystem Services, RIOS=Resource Investment Optimization System; $\mathrm{RC}+\mathrm{RB}=$ conceptual networks + Bayesian Networks.

\begin{tabular}{|c|c|c|c|c|c|c|}
\hline \multirow[b]{2}{*}{ Aproximación } & \multicolumn{6}{|c|}{ Criterios de evaluación } \\
\hline & $\begin{array}{c}\text { Evaluaciór } \\
\text { de un } \\
\text { conjunto } \\
\text { de SE }\end{array}$ & Metodología de análisis & $\begin{array}{l}\text { Representación } \\
\text { espacialmente } \\
\text { explícita de los } \\
\text { resultados obtenidos }\end{array}$ & $\begin{array}{l}\text { Combinación de } \\
\text { valoración económica } \\
\text { y ecológica de la } \\
\text { provisión de SE }\end{array}$ & $\begin{array}{c}\text { Relación entre } \\
\text { la provisión de } \\
\text { SE y el bienestar } \\
\text { humano }\end{array}$ & $\begin{array}{c}\text { Evaluación del efecto } \\
\text { del cambio climático } \\
\text { sobre la provisión } \\
\text { de SE }\end{array}$ \\
\hline ECOSER & Sí & $\begin{array}{l}\text { Modelos, reglas e } \\
\text { indicadores }\end{array}$ & Sí & No & Sí & Sí \\
\hline InVEST & Sí & $\begin{array}{l}\text { Funciones de } \\
\text { producción }\end{array}$ & Sí & Sí & Sí & Sí \\
\hline ARIES & Sí & $\begin{array}{c}\text { Ontologías y Redes } \\
\text { Bayesianas }\end{array}$ & Sí & No & Sí & Sí \\
\hline RIOS & Sí & $\begin{array}{l}\text { Ecuaciones e índices } \\
\text { sumado a funciones de } \\
\text { producción }\end{array}$ & Sí & Sí & Sí & Sí \\
\hline $\mathrm{RC}+\mathrm{RB}$ & Sí & $\begin{array}{l}\text { Redes conceptuales y } \\
\text { Redes Bayesianas }\end{array}$ & $\begin{array}{c}\text { No } \\
\text { (A futuro) }\end{array}$ & No & Sí & Sí \\
\hline
\end{tabular}


estructuras formales que describen un dominio conceptual que suele consistir en un grupo de axiomas que definen conceptos y relaciones entre conceptos (Wand et al. 1999)) con RB, similar a RC+RB. Una diferencia es que en ARIES las técnicas de inteligencia artificial examinan los datos de origen y seleccionan del grupo de ontologías aquellos modelos que mejor representan la situación bajo estudio (Villa et al. 2009).

\section{Representación espacialmente explícita de los resultados obtenidos}

Encontrasteconel restodelasaproximaciones, este aspecto no fue considerado por $\mathrm{RC}+\mathrm{RB}$. Si bien bajo $\mathrm{RC}+\mathrm{RB}$ los resultados obtenidos son factibles de ser representados espacialmente, esto no resultará sencillo ya que se deberá reducir la escala de análisis (i.e., pasar de una escala de región a una escala de lote) de las variables de entrada.

\section{Combinación de evaluación económica y ecológica de la provisión de SE}

InVEST tiene como salida tanto los flujos de SE expresados en unidades biofísicas como ponderados por su valor económico y expresados en dólares. RIOS proporciona los beneficios en términos económicos mientras que ECOSER, ARIES y RC+RB están únicamente enfocados a la evaluación ecológica.

\section{Relación entre la provisión de SE y el bienestar humano}

InVEST, RIOS, ARIES y ECOSER evalúan el bienestar humano resultante de un nivel adecuado de provisión de SE. Si bien este aspecto no fue considerado de forma explícita en $\mathrm{RC}+\mathrm{RB}$, es posible hacer inferencias al respecto a partir de los resultados obtenidos.

\section{Evaluacióndelefectodelcambioclimáticosobre la provisión de $S E$}

Tanto InVEST como ARIES pueden explorar los cambios en distintas variables climáticas para luego predecir cambios en la provisión de SE en función de un conjunto de escenarios climáticos futuros. ECOSER calcula la vulnerabilidad socioecológica en base a escenarios de cambio generados o adoptados por el usuario. Por lo tanto, se pueden aplicar escenarios de cambio de uso/cobertura de la tierra. RIOS también presenta la posibilidad de generar escenarios de uso de la tierra.
$\mathrm{RC}+\mathrm{RB}$ podría incorporar en sus variables de entrada predicciones climáticas provistas por el Panel Intergubernamental para el Cambio Climático para generar escenarios climáticos y productivos futuros.

La complementariedad entre aproximaciones sugiere la posibilidad de utilizarlas conjuntamente con el objetivo de cubrir distintos aspectos de la evaluación de la provisión de SE (Tabla 1). En particular, $\mathrm{RC}+\mathrm{RB}$ podría ser integrada en una o más de las restantes herramientas. Por ejemplo, ECOSER incorpora la opinión de expertos desde un enfoque determinista $\mathrm{y}$ la consideración de RB mejoraría mucho este aspecto. En este caso, será necesario que el futuro usuario decida, en base a los criterios de evaluación aquí expuestos, cuáles son aquellas aproximaciones que mejor se adaptan a sus necesidades de estudio, como la información de entrada requerida, sector (público o privado) de interés de los resultados, contexto de investigación, tipo de resultados a obtener (e.g., ecológicos, económicos, espaciales, probabilísticos), entre otros. Debido a que estas aproximaciones continuarán con su proceso de desarrollo, los beneficios a obtener serán más enriquecedores cuando los participantes tengan una influencia directa sobre el proceso de generación o evolución de las mismas (Tuler and Webler 1999), la información utilizada y aplicada sea considerada relevante por los participantes (Webler et al. 2001), y el lenguaje y los conceptos utilizados en el debate propicien una buena comunicación entre los desarrolladores y los futuros usuarios (Rowe and Frewer 2004).

\section{CONCLUSIONES}

En este trabajo se evaluó un nuevo marco de análisis de la provisión de SE. Este marco se caracteriza por proponer criterios claros y explícitos mediante la inferencia bayesiana, además de permitir la incorporación de diferentes fuentes de información (i.e., bibliografía, conocimiento experto, bases de datos ambientales y productivas). Además, no sólo presenta ventajas (e.g., manejo de la incertidumbre inherente a los sistemas, incorporación de conocimiento experto) y desventajas (e.g., no incorpora la representación espacial de los resultados) frente a otras herramientas de evaluación de SE, sino también la posibilidad de complementarse con ellas en base a las características aquí resumidas. Finalmente, este marco de análisis es un primer paso para 
el desarrollo de una herramienta amigable que permita a los productores y asesores agropecuarios complementar el diseño de sistemas agrícolas con estimaciones de los efectos sobre el ambiente y sus futuras producciones.

AgRADECIMIENTOS. Este trabajo fue financiado por el Consejo Nacional de Investigaciones Científicas y Técnicas (PIP 555), por la
Agencia Nacional de Promoción Científica (PICT 1559) y por la Universidad de Buenos Aires (UBACYT 20020110100196). Florencia Rositano fue beneficiaria de una beca doctoral CONICET. Agradecemos a V. Logegaray, P. Graff y C. Gelabert así como a Pedro Laterra (editor asociado) y a dos revisores anónimos por sus comentarios y sugerencias que enriquecieron sustancialmente el manuscrito original.

\section{REFERENCIAS}

Ascough II, J. C., H. R. Maier, J. K. Ravalico, and M. W. Strudley. 2008. Future research challenges for incorporation of uncertainty in environmental and ecological decision-making. Ecol Model 219:383-399.

Bressan, G. M., V. A. Oliveira, E. R. Hruschka J. R., and M. C. Nicoletti. 2009. Using Bayesian networks with rule extraction to infer the risk of weed infestation in a corn-crop. Eng Appl Artif Intel 22:579-592.

Busch, M., A. La Notte, V. Laporte, and M. Erhard. 2012. Potentials of quantitative and qualitative approaches to assessing ecosystem services. Ecol Indic 21:89-103.

Chen, S. H., and C. A. Pollino. 2012. Good practice in Bayesian network modelling. Environ Modell Softw 37:134145.

Cornelissen, A. M. G., J. Van Den Berg, W. J. Koops, and U. Kaymak. 2003. Elicitation of expert knowledge for fuzzy evaluation of agricultural production systems. Agric Ecosyst Environ 95:1-18.

Dlamini, W. M. 2010. A Bayesian belief network analysis of factors influencing wildfire occurrence in Swaziland. Environ Modell Softw 25:199-208.

Dorner, S., J. Shi, and D. Swayne. 2007. Multi-objective modelling and decision support using a Bayesian network approximation to a non-point source pollution model. Environ Modell Softw 22:211-222.

Ellison, S. L. R., S. Gregory, and W. A. Hardcastle. 1998. Quantifying uncertainty in qualitative analysis. The Analyst 123:1155-1161.

Grêt-Regamey, A., S. H. Brunner, J. Altwegg, and P. Bebi. 2012. Facing uncertainty in ecosystem services-based resource management. J Environ Manage 127:145-154.

Haines-Young, R. 2011. Exploring ecosystem service issues across diverse knowledge domains using Bayesian Belief Networks. Prog Phys Geogr 35:681-699.

Kareiva, P., H. Tallis, T. H. Ricketts, G. C. Daily, and S. Polasky. 2011. Natural Capital: Theory and Practice of Mapping Ecosystem Services. Oxford University Press, Oxford.

Lamanda, N., S. Roux, S. Delmotte, A. Merot, B. Rapidel, et al. 2012. A protocol for the conceptualisation of an agroecosystem to guide data acquisition and analysis and expert knowledge integration. Eur J Agr 38:104-116.

Landuyt, D., S. Broekx, R. D’Hondt, G. Engelen, J. Aertsens, and P. L. M. Goethals. 2014. A review of Bayesian belief networks in ecosystem service modeling. Environ Modell Softw 46:1-11.

Laterra, P., P. Barral, A. Carmona, and L. Nahuelhual. 2015. ECOSER. Protocolo colaborativo de evaluación y mapeo de servicios ecosistémicos y vulnerabilidad socio-ecológica para el ordenamiento territorial. Documento Introductorio. Versión 2.0. Ediciones INTA, Colección Investigación, Desarrollo e Innovación No. 99. Santa Rosa, La Pampa. ISSN impreso 0325-2132. Pp. 56.

Laterra, P., P. Barral, A. Carmona, and L. Nahuelhual. 2016. Focusing conservation efforts on ecosystem service supply may increase vulnerability of socio-ecological systems. PLoS ONE 11(5):e0155019. doi:10.1371/journal.pone.0155019.

López Puga, J., J. García García, L. De La Fuente Sánchez, and E. I. De La Fuente Solana. 2007. Las redes bayesianas como herramientas de modelado en psicología. An Psicol 23:307-316.

Low Choy, S., R. O'Leary, and K. Mengersen. 2009. Elicitation by design in ecology: using expert opinion to inform priors for Bayesian statistical models. Ecology 90:265-277.

Müller, F. 2005. Indicating ecosystem and landscape organisation. Ecol Indic 5:280-294.

Pollino, C. A., O. Woodberry, A. Nicholson, K. Korb, and B. T. Hart. 2007. Parameterisation and evaluation of a Bayesian network for use in an ecological risk assessment. Environ Modell Softw 22:1140-1152.

Rapidel, B., C. Defèche, B. Traoré, J. Lancon, and J. Wery. 2006. In field development of a conceptual model for crop functioning and management: a case study on cotton in Southern Mali. Eur J Agr 24:304-315.

Rositano, F., and D. O. Ferraro. 2014. Ecosystem services provided by agroecosystems: A qualitative and quantitative assessment of this relationship in the Pampa region, Argentina. Environ Manage 53(3):606-619.

Rowe, G., and L. J. Frewer. 2004. Evaluating public-participation exercises: a research agenda. Sci Technol Hum Val 29:512-557.

Smith, R. I., J. M. C. P. Dick, and E. M. Scott. 2011. The role of statistics in the analysis of ecosystem services. Environmetrics 22:608-617.

Tallis, H. T., T. Ricketts, A. D. Guerry, S. A. Wood, R. Sharp, et al. 2013. InVEST 2.6.0 User's Guide. The Natural Capital Project, Stanford. 
Tuler, S., and T. Webler. 1999. Voices from the forest: what participants expect of a publication participation process. Soc Natur Resour 12:437-453.

Uusitalo, L. 2007. Advantages and challenges of Bayesian networks in environmental modelling. Ecol Model 203: 312-318.

Van Der Gaag, L., and E. Helsper. 2002. Experiences with modelling issues in building probabilistic networks. Pp. 21-26 in: A. Gómez-Pérez and V. R. Benjamins (eds.). Knowledge Engineering and Knowledge Management: Ontologies and the Semantic Web. Springer Verlag, Berlin.

Vihervaara, P., T. Kumpula, A. Tanskanen, and B. Burkhard. 2010. Ecosystem services - A tool for sustainable management of human-environment systems. Case study Finnish Forest Lapland. Ecol Complexity 7:410-420.

Villa, F., M. Ceroni, K. Bagstad, G. Johnson, and S. Krivov. 2009. ARIES (ARtificial Intelligence for Ecosystem Services): a new tool for ecosystem services assessment, planning, and valuation. BioEcon 1-10.

Villa, F., K. J. Bagstad, B. Voigt, G. W. Johnson, R. Portela, et al. 2014. A methodology for adaptable and robust ecosystem services assessment. PLoS ONE 9(3):e91001.

Vogl, A., H. Tallis, J. Douglass, R. Sharp, F. Veiga, et al. 2013. Sistema de Optimización de Inversión de Recursos. Introducción y Documentación teórica. Pp. 26.

Walker, W. E., P. Harremoes, J. Rotmans, J. P. Van Der Sluijs, M. B. A. Van Asselt, et al. 2003. Defining uncertainty. A conceptual basis for uncertainty management in model-based decision support. Integr Assess 4(1):5-17.

Wand, Y., V. C. Storey, and R. Weber. 1999. An ontological analysis of the relationship construct in conceptual modelling. Acm T Database Syst 24:494-528.

Webler, T., S. Tuler, and R. Krueger. 2001. What is a good public participation process? Five perspectives from the public. Environ Manage 27:435-450. 